\title{
Integrated bees algorithm and artificial neural network to propose an efficient controller for active front steering control of vehicles
}

\author{
B. Aalizadeh ${ }^{1}$ and A. Asnafi ${ }^{2, *}$ \\ ${ }^{1}$ Department of Mechanical Engineering, Sama Technical and Vocational Training \\ College, Islamic Azad University, Gachsaran Branch Gachsaran, Iran. \\ 2,*Hydro-Aeronautical Research Center, Shiraz University, Shiraz, 71348-13668, Iran. \\ E-mail: asnafi@shirazu.ac.ir \\ Phone: +98-713-36133771, Fax: +98-71-32290296.
}

\begin{abstract}
In this paper, a new optimal adaptive controller for the active front steering control of a vehicle is proposed. Due to the availability and applicability of proportional-integrator-derivative (PID) controllers, this controller is picked up; but, to overcome its limitations, two optimization and adaptation schemes are employed. The reference transfer function between the yaw rate of a typical vehicle and its steering angle is derived. The actual dynamics is simulated using CarSim toolbox of MATLAB. Best vehicle handling was aimed to be reached for three famous driving manoeuvres by proposing an efficient but economic controller. An optimization is done on the PID coefficients for a specific road condition using the honey bees' algorithm, and then a two-layer artificial neural network is trained using the back propagation learning rule to adjust the controller coefficients for any arbitrary road conditions. The uncontrolled, desired, optimized controlled, and optimized plus trained controlled yaw rate of vehicles are drawn for three manoeuvers and three road conditions. The integral of squared error between the desired and actual trajectories for different manoeuvers and road conditions are evaluated and compared between each other. The performance of the proposed PID controller that was optimized by Bees Algorithm and trained by a neural network was proved. It is noted that the optimized PID controller is good for all road conditions but not excellent. The more we move away from the reference road (in which, the optimization is done), the error will be larger. Thus for other conditions, using the artificial neural network can help decrease the error significantly.
\end{abstract}

Keywords: Active front steering control; bees algorithm; artificial neural network; vehicle handling.

\section{INTRODUCTION}

Vehicle handling and stability have great effects on the comfort and safety of travel. Since a vehicle may encounter unpredictable and uncertain parameters such as air and road conditions, design of a quick, precise, adaptive, and of course economic controller can help the driver to improve the driving in real time. The performance of steering system plays a vital role in the handling and the yaw stability control of any road vehicle. The vehicle stability may be affected by some expected and unexpected factors including the vehicle's structure and parameters, the initial operation of the vehicle, tire steer- 
ing angle, road conditions, side wind force, tire pressure loss or vehicle driving situations [1].Under these almost unpredictable conditions, the driver may not respond within a very short time; therefore, it is necessary to develop secure automatic control approaches. There are several methods to control the yaw rate of a vehicle such as direct yaw moment control [2], four wheel steering system control [3], active differential braking [4], semi active steering system [5, 6], and active front steering (AFS) control [6].

The latter is one of the efficient methods to solve the above mentioned problem that is based on stabilizing the yaw rate of a vehicle just by manipulating the wheel steering angle. Simply put, it tries to compensate an additional angle to the wheel steering angle that improves the vehicle's manoeuvrability, stability and path tracking [6].This compensation has been done in literature using several types of controllers. The fuzzy logic controller was employed to improve the yaw rate evolution for a four-wheel differential (4WD) [7]. The performance of fuzzy logic controllers was studied and discussed to control the yaw rate and side slip angle of vehicles [8]. Canale, Fagiano [9] designed a robust controller to control the yaw rate of vehicles by means of rear active differential techniques. The presented controller did not work properly for unpredictable and varying conditions and showed good performance for some specific ranges and conditions. $\mathrm{Wu}, \mathrm{Zhu}[10]$ constructed a reference model for a vehicle via a neural network using back propagation of error as the learning algorithm, and a fuzzy controller optimized by genetics algorithm for active steering control. This control arrangement was applied to a four-wheel steering system at some distinctive conditions. Integrated yaw moment and active front steering control was employed by $\mathrm{Wu}, \mathrm{Zhao}$ [11] to design a fuzzy logic controller for yaw stability improvement of a vehicle. Similar to previous mentioned works, their controller was designed for some determined road frictions and conditions.

Most of the active steering control systems which have been recommended in literature used complicated and sometimes non-economic controller elements for specific dynamic models, and assumed constant road and parameter conditions (see for example [12-14]). Due to unpredictable and unexpected parameter changes, a simple applicable but efficient controller must be proposed in order to adjust itself with these variations. An advisable strategy to design such controllers is to combine both the adaptation and optimization for the parameter selection of these controllers.

In this paper, using the integrated honey bee's optimization algorithm and a two-layer artificial neural network estimator, a simple economic PID but efficient controller is proposed. In fact, joint use of optimization and adaptation over one of the most practical and industrial controller i.e. the PID controller presents a quick, precise, and economic scheme for vehicle handling. The procedure is done by first optimizing the coefficients of a PID controller via Bees Algorithm for a specific road condition, and then tuning the coefficients for any unpredictable conditions using the neural network. Combination of these above methods to design an efficient but simply available active steering controller with acceptable performance in different road conditions has been less studied in literature. One of the relatively relevant articles in this context is the article written by Zhuo-Ming, Yun-Xia [15] who applied the artificial bee's colony algorithm to optimize synaptic weights of an Artificial Neural Network (ANN).

\section{METHODS AND MATERIALS}

\section{Vehicle model description}

In this section, both the reference and actual models for our system will be introduced. The reference model produces the reference signal which is used in the control 
simulation scheme. With respect to Figure 1, the reference model is a $2 \mathrm{D}$ reduction of a real 3D vehicle whose rear and front axles were replaced by two wheels. In a real vehicle, the best condition for minimized slipping and optimal handling occurs when the wheels rotate about a point. This introduced model can satisfy this optimal condition for desired handling simply. One of the advantages of this model is that the response of vehicles under step input of steering is a circle and consequently the yaw rate is a constant value $[16,17]$.

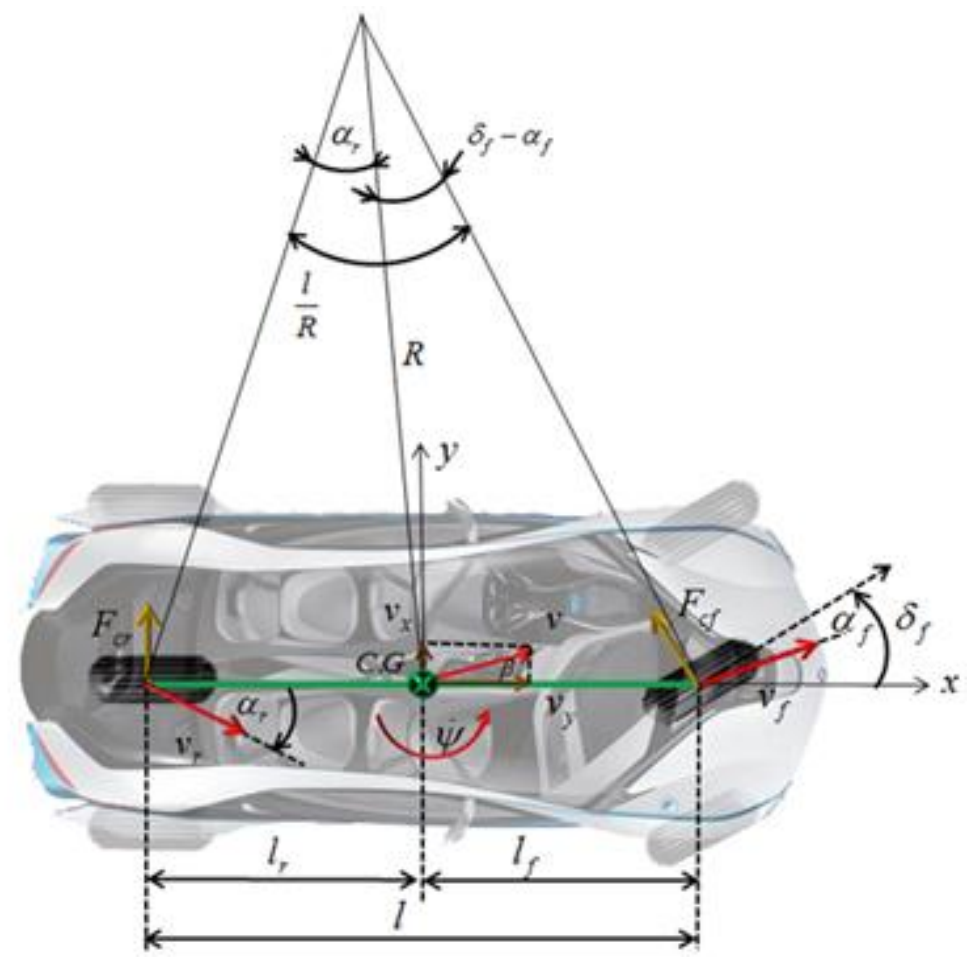

Figure 1. The schematic diagram of the reference model.

With reference to Figure 1, the $2 \mathrm{D}$ governing equations of motion become $[16,17]$ :

$$
\begin{gathered}
m\left(\dot{v}_{y}+\dot{\psi} v_{x}\right)=F_{c r}+F_{c f} \cos \delta_{f} \\
m\left(-\dot{\psi} v_{y}\right)=-F_{c f} \sin \delta_{f} \\
I_{z} \ddot{\psi}=l_{f} F_{c f}-l_{r} F_{c r}
\end{gathered}
$$

where $\mathrm{m}$ is the total mass of car, $v_{y}$ and $v_{x}$ are velocity at $y$ and $x$ directions respectively, $F_{c r}$ and $F_{c f}$ are the lateral forces of rear and front wheels, $\delta_{f}$ is the steering angle of front wheel, $I_{z}$ is the mass moment of inertia and $\psi$ is the car yaw angle. $l_{f}$ and $l_{r}$ are the distances between front/rear wheel and the centre of mass point, $l$ is the distance between the rear and front wheels, $\alpha_{f}$ and $\alpha_{r}$ are the lateral slip angles of front and rear wheel respectively.

Assuming the constant cornering stiffness for rear and front tires led someone to rewrite the lateral forces as $[16,17]$ : 


$$
\begin{gathered}
F_{c f}=C_{f} \alpha_{f} \Rightarrow F_{c f}=C_{f}\left(\beta+\frac{\dot{\psi} l_{f}}{v_{x}}-\delta_{f}\right) \\
F_{c r}=C_{r} \alpha_{r} \Rightarrow F_{c r}=-C_{r}\left(\beta-\frac{\dot{\psi} l_{r}}{v_{x}}\right)
\end{gathered}
$$

where $C_{f}, C_{r}$ are the cornering stiffness of the front and rear wheels respectively, and $\beta$ is the sideslip angle of the car. It is assumed that the vehicle travels with a constant velocity along the $x$ direction; therefore, by choosing $\beta$ and $\mathrm{d} \psi / \mathrm{dt}$ as the state variables, one can simply reach to the such following state space equation between $\delta_{f}$ and defined state variables:

$$
\left[\begin{array}{c}
\dot{\beta} \\
\ddot{\psi}
\end{array}\right]=\left[\begin{array}{cc}
-\frac{\left(C_{f}+C_{r}\right)}{m v_{x}} & -1-\frac{\left(l_{f} C_{f}-l_{r} C_{r}\right)}{m v_{x}^{2}} \\
-\frac{\left(l_{f} C_{f}-l_{r} C_{r}\right)}{I_{z}} & -\frac{\left(l_{f}^{2} C_{f}+l_{r}^{2} C_{r}\right)}{m v_{x}^{2}}
\end{array}\right]\left[\begin{array}{c}
\beta \\
\dot{\psi}
\end{array}\right]+\left[\begin{array}{c}
\frac{C_{f}}{m v_{x}} \\
\frac{l_{f} C_{f}}{I_{z}}
\end{array}\right] \delta_{f}
$$

A simple calculation results in the transfer function between the yaw rate and the steer angle of the front wheel as $[16,17]$ :

$$
\frac{\dot{\psi}}{\delta_{f}}=\frac{A_{1} s+A_{2}}{B_{1} s^{2}+B_{2} s+B_{3}}
$$

where $s$ is the Laplace operator and the coefficients $A_{1}, A_{2}, B_{1}, B_{2}$, and $B_{3}$ are:

$$
\begin{aligned}
& A_{1}=m v_{x} l_{f} C_{f} \\
& A_{2}=L C_{f} C_{r} \\
& B_{1}=m v_{x} I_{z} \\
& B_{2}=I_{z}\left(C_{f}+C_{r}\right)+m\left(l_{f}^{2} C_{f}+l_{r}^{2} C_{r}\right) \\
& B_{3}=\frac{C_{f} C_{r}}{v_{x}}\left(l_{f}+l_{r}\right)^{2}\left[1+\frac{m v_{x}^{2}\left(l_{r} C_{r}-l_{f} C_{f}\right)}{C_{f} C_{r}\left(l_{f}+l_{r}\right)^{2}}\right]
\end{aligned}
$$

With respect to Figure 1, it can be shown that:

$$
\delta_{f}-\alpha_{f}+\alpha_{r}=\frac{l}{R} \rightarrow \delta_{f}=\alpha_{f}-\alpha_{r}+\frac{l}{R}
$$

Also, by rewriting the Eq. (4) and Eq. (5), we have:

$$
\alpha_{f}=\frac{F_{c f}}{C_{f}}=\frac{m_{f}}{C_{f}} \frac{v^{2}}{R}
$$




$$
\alpha_{r}=\frac{F_{c r}}{C_{r}}=\frac{m_{r}}{C_{r}} \frac{v^{2}}{R}
$$

where $m_{f}$ and $m_{r}$ are the equivalent front and rear masses, respectively. It is noted that these masses are computed in static situation of the vehicle. Substituting Eq. (10) and Eq. (11) to Eq. (9), one can reach to:

$$
\begin{aligned}
& \delta_{f}=\frac{l}{R}+\left(\frac{m_{f}}{C_{f}}-\frac{m_{r}}{C_{r}}\right) \frac{v^{2}}{R} \\
& =\frac{l}{R}+\left(\frac{m_{f}}{C_{f}}-\frac{m_{r}}{C_{r}}\right) a_{y} \\
& =\frac{l}{R}+K_{u} a_{y}
\end{aligned}
$$

where $a_{y}$, is the lateral acceleration and $K_{u}$ is known as the under steering coefficient, with the such following relation $[16,17]$ :

$$
K_{u}=\frac{m_{f}}{C_{f}}-\frac{m_{r}}{C_{r}}=\left(\alpha_{f}-\alpha_{r}\right) \frac{R}{v^{2}}
$$

Finally, using Eqs. (10) - (13), one can rearrange the transfer function between the yaw rate of vehicle and steering angle Eq. (7) as:

$$
\dot{\psi}=\frac{v \delta_{f}}{l+k_{u} v^{2}}
$$

Equation (14) prepares the reference signal for our control simulation arrangement while in this paper, a virtual but completely efficient software is used to model the actual plant. CarSim, as one of the MATLAB Simulink toolbox is used to model an actual vehicle with real and different road conditions and other true situations. In literature, many works have used this toolbox as a reliable simulator for the vehicle behaviour investigation under real and true conditions (see for example, [8]). CarSim has been proven as a powerful software package to simulate vehicle response with respect to control inputs and used in numerous studies (see for example $[18,19]$ ).

Table 1 shows the parameter values that are used in both reference and actual models in this paper. In this table, $R_{w}$ is the nominal radius of the wheel, $I_{w}$ is the mass moment of inertia of the wheel, $h$ is the height of the centre of gravity of suspended vehicle, and $l_{w}$ is the width between right and left wheels.

Table 1. The parameters of the vehicle used in our simulations.

\begin{tabular}{cccccc}
\hline$m$ & $1530(\mathrm{~kg})$ & $l_{f}$ & $1.14(\mathrm{~m})$ & $R_{w}$ & $0.31(\mathrm{~m})$ \\
\hline$I_{z}$ & 4607.5 & $l_{r}$ & $1.64(\mathrm{~m})$ & $I_{w}$ & $0.9\left(\mathrm{~kg} \cdot \mathrm{m}^{2}\right)$ \\
$v$ & $\begin{array}{c}\left(\mathrm{kg} \cdot \mathrm{m}^{2}\right) \\
v(\mathrm{~km} / \mathrm{h})\end{array}$ & $l_{w}$ & $1.55(\mathrm{~m})$ & $H$ & $0.52(\mathrm{~m})$ \\
\hline
\end{tabular}




\section{Controller design pattern}

The conventional PID controllers cannot be used as efficient organizers to reach the desired behaviour for such complicated problems. In fact, the controller must work properly and instantly for different roads and driving conditions in real time. This brings us to the use of an optimized adaptive controller. On the other hand, due to the generality, applicability, widespread use, and simplicity of PID controller, these organizers are the first candidates for manipulation of common industrial applications. In this research, the parameters of a conventional PID controller, i.e. $K_{p}, K_{d}$ and $K_{i}$ must be optimized and adjusted during the control process at the same time. Here both the BA (Bees Algorithm) and ANN (Artificial Neural Network) are jointly employed to modify the conventional PID controller and overcome the demanded circumstances. As can be found in literature, the BA is a random search algorithm which is inspired from the honey bee colonies' manner for gathering the flower patches and foraging strategy in nature [20]. In harvesting season, honey bees try to obtain the best nectars with minimum energy consumption. In this process, first the scout bees begin to search for promising flower patches by randomly moving from one patch to another [20]. If the nectar of a patch of flowers satisfies the certain fitness threshold, its information including the distance to the hive, its relative direction, and its quality are shared with other colony bees via a mysterious manoeuver which is known as the "Waggle dance" [21]. This information helps the colony to find the efficient patches, i.e. those with higher quality nectars and lower energy consumption for gathering, harvesting, and transportation [21].

An optimization algorithm was first introduced based on this natural procedure by [22]. The algorithm has been successfully applied to many optimization problems in literature such as design of mechanical components [23], multi-objective optimization [24], neural network training t Pham, Soroka [25], and image analysis [26], etc. The general operation flowchart of this algorithm is [27]:

- Initialise population with random solutions.

- Evaluate fitness of the population.

- While (stopping criterion not met),

- Form new population.

- Select sites for neighbourhood search.

- Recruit bees for selected sites (more bees for best e-sites) and evaluate fitness.

- Select the fittest bee from each patch.

- Assign remaining bees to search randomly and evaluate their fitness.

- End while.

Note also that the capability of the BA as a powerful tool for optimization problems has been proved in many researches (see for example [28-30]). In the simulation and with respect to the above flowchart, first a set of initial values for PID coefficients were chosen randomly. This set constructed the initial population of $\mathrm{n}$ scout bees. After that, the integral of the squared error signal as the fitness function was evaluated for each bee (initial value of the PID coefficients). The best m numbers of bees were selected and the others were removed. The selected bees were divided to two groups of elite (e) and ordinary (m-e) bees. After the radius of neighbourhood (ngh) for the patches was selected, ne numbers of bees were recruited to check the fitness criterion and find the best solution in elite sites, while other ns bees were employed to search remaining selected but ordinary sites. The procedure was repeated for remaining (n-m) scout bees to 
search the space randomly until a stopping criterion has been reached. In Table 2, someone can find the values of parameters used in the algorithm.

Table 2. The values of parameters that are used in our Bees Algorithm.

\begin{tabular}{|c|c|c|}
\hline Description & $\begin{array}{l}\text { Notation } \\
\text { (parame- } \\
\text { ter) }\end{array}$ & Value \\
\hline The initial population of scout bees & $\mathrm{n}$ & 100 \\
\hline The best numbers of bees & $\mathrm{m}$ & 15 \\
\hline Number of elite patches & $\mathrm{e}$ & 5 \\
\hline Number of bees were recruited to check elite sites & ne & 20 \\
\hline $\begin{array}{l}\text { Number of bees were employed to check ordinary se- } \\
\text { lected sites }\end{array}$ & ns & 10 \\
\hline $\begin{array}{l}\text { Neighbourhood of the first parameter of PID control- } \\
\text { ler }\left(K_{p}\right)\end{array}$ & $\mathrm{Ngh}_{1}$ & 0.5 \\
\hline $\begin{array}{l}\text { Neighbourhood of the second parameter of PID con- } \\
\text { troller }\left(\mathrm{K}_{\mathrm{i}}\right)\end{array}$ & $\mathrm{Ngh}_{2}$ & 0.000001 \\
\hline $\begin{array}{l}\text { Neighbourhood of the third parameter of PID control- } \\
\text { ler }\left(\mathrm{K}_{\mathrm{d}}\right)\end{array}$ & $\mathrm{Ngh}_{3}$ & 0.000001 \\
\hline The stopping criterion (number of iteration) & - & 1000 \\
\hline
\end{tabular}

Using the above pointed procedure, an optimized set for the coefficient of PID controller was obtained. Here, to reach a controller that can be applied for different road conditions, an estimation and adaptation procedure using the artificial ne ural networks is also employed. As it can be seen in many works, an artificial neural network is an imitation of making a decision with respect to previous trained behaviours and data in the biological human brain [31-33]. After an ANN has been trained by some defined and determined input/output signals, it may decide about a suitable response due to an unknown input [34]. The efficiency and correctness of this method has been discussed in many applications including control systems. See for example [34-36].

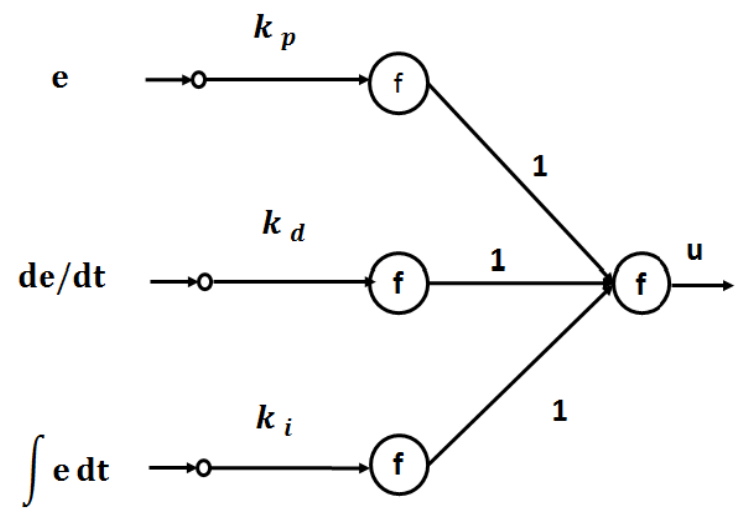

Figure 2. The architecture of the network used in our simulation.

Figure 2 shows the architecture of a two-layer feed-forward neural network controller that is used in our simulation. In this model, all the activation functions (f) are assumed to be linear while the layer weights are considered to be one. With reference to this architecture, the control signal $u$ becomes: 


$$
u=k_{p} \times e(t)+k_{d} \times \frac{d e}{d t}+k_{i} \times \int e(t) d t
$$

During the simulation and in each iteration, the error between the output of the plant and the output of the desired model is computed via the following relation:

$$
e(n)=Y_{r e f}-y(n)
$$

Now, the cost function is defined as:

$$
E=1 / 2\left(e^{2}(n)\right)
$$

To minimize the cost function Eq. (17), the back propagation method is used. The method was first introduced by Rojas [37], and then used in neural networks as a learning algorithm in [38]. Here, this method is used to update the layer weights iteratively to reach an optimal value for the desired cost function. Unfortunately, we do not have information about the hidden layers but we can compute how fast the error changed as a hidden action was changed. Therefore, to train a network, the error derivative with respect to hidden action can be used instead of desired action itself. These concepts of back propagation algorithm together with the delta learning rule $[39,40]$ are used to compute layer weights for minimizing the cost function $E$, Eq. (10) in this study. Based on above methods, the layer weight variation in each iteration must be proportional to the minus gradient of the cost function with respect to that layer weight [31] i.e.:

$$
\Delta w_{i} \propto-\frac{\partial E}{\partial w_{i}} \stackrel{\text { Chain rule }}{=}-\frac{\partial E}{\partial e(n)} \frac{\partial e(n)}{\partial y(n)} \frac{\partial y}{\partial u} \frac{\partial u}{\partial w_{i}}
$$

where $w_{i}$ is the weight of $\mathrm{i}^{\text {th }}$ layer. A simple calculation leads someone to reach the such following relation for layer weigh changing rule:

$$
\Delta w_{i}=\eta e(n) \frac{\partial u}{\partial w_{i}}
$$

where $\eta$ is the coefficient of training. This coefficient must be selected such that both the stability of iterations and speed of training will be satisfied. In this study, $\eta=0.6$ satisfies both conditions; therefore, the updating law of layer weights becomes:

$$
w_{i}(n+1)=w_{i}(n)+\eta e(n) \frac{\partial u}{\partial w_{i}}
$$

Similarly, the updating rules for PID parameters become:

$$
k_{p}(n+1)=k_{p}(n)+\eta \times e(n) \times e(n)
$$




$$
\begin{aligned}
& k_{i}(n+1)=k_{i}(n)+\eta \times e(n) \times \int e(n) d t \\
& k_{d}(n+1)=k_{d}(n)+\eta \times e(n) \times \frac{d e(n)}{d t}
\end{aligned}
$$

Briefly, the coefficients of the designed and proposed PID controller are first optimized by the Bees Algorithms for a conventional road condition, i.e. when the coefficient of friction is equal to 1 . After that, using a two-layer artificial neural network, the optimized coefficients are tuned for other road conditions. In the following section, the efficiency and effectiveness of the controller for three types of road conditions will be justified in real time for some famous vehicle manoeuvers.

\section{RESULTS AND DISCUSSION}

In this section, the correctness and efficiency of the designed and proposed controller is investigated to track three famous manoeuvres i.e. J-turn, fishhook, and change lane. Figure 3 illustrates these three famous manoeuvres schematically. The steering angles of the front wheel regarding to these manoeuvres with respect to time are drawn in Figure 4. With reference to this figure, as a driver decides to change its lane, he/she applies a positive/negative steering angle. Once the lane is changed, a counter angle is necessary to reorient the vehicle in the new lane. In both J-turn and fishhook manoeuvres, a non-zero steering angle is necessary to change the car direction. A single-sided steering angle is used for the former while a double-sided one is employed for the latter.

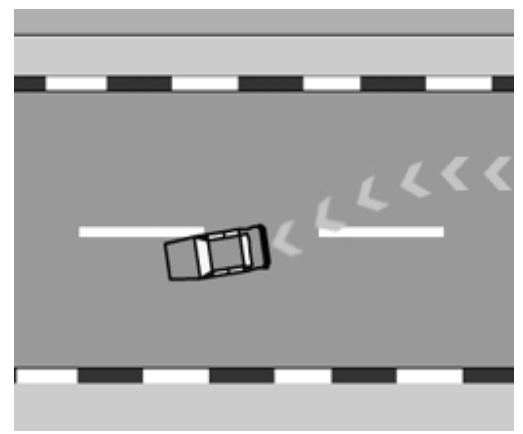

(a)

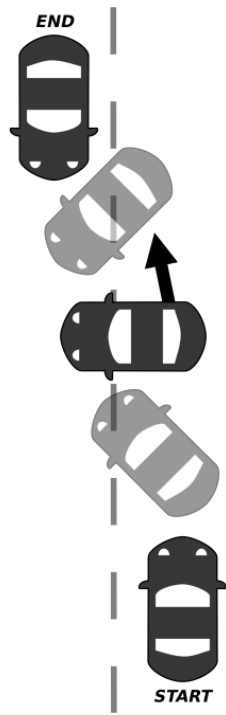

(b)

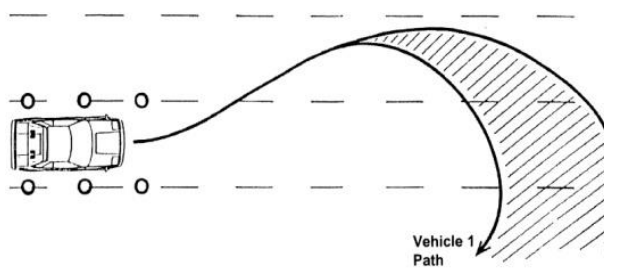

(c)

Figure 3. Schematic diagram of a: change-lane, b: J-turn and c: fishhook manoeuvres.

The coefficient of friction as an index for the road condition is selected and varied from an almost regular road to an icy one. To have a better estimation and judgement, the proposed PID controller in the previous section, before and after application of the artificial neural network is employed to manipulate the system. In Figure 5, the desired, uncontrolled, and controlled yaw rate of the pointed vehicle for the change lane manoeuvres at different road conditions are drawn. 


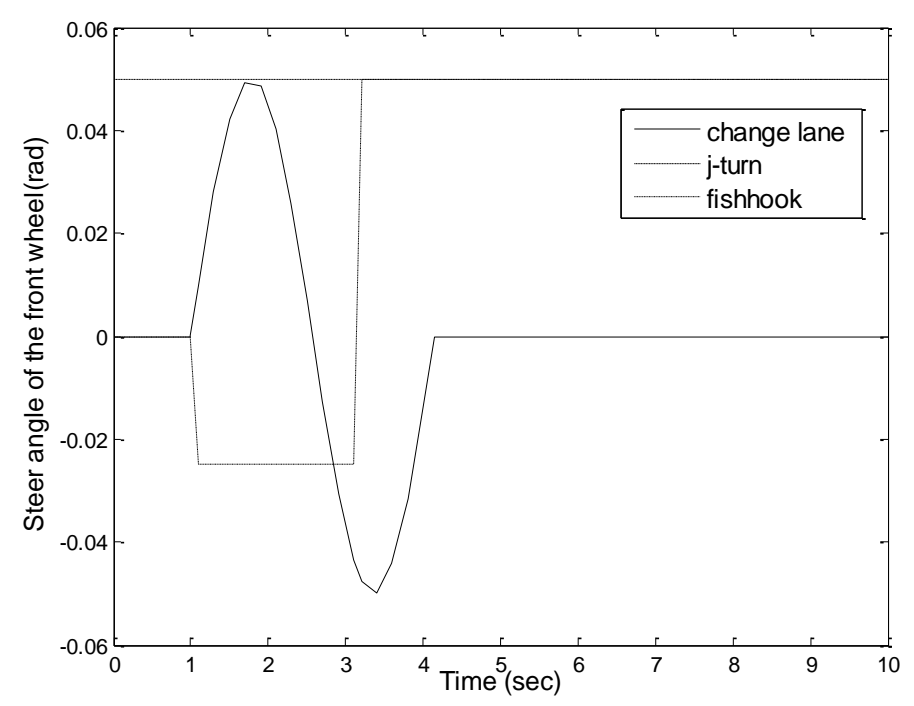

Figure 4. The steering angle of the front wheel in each manoeuvre.

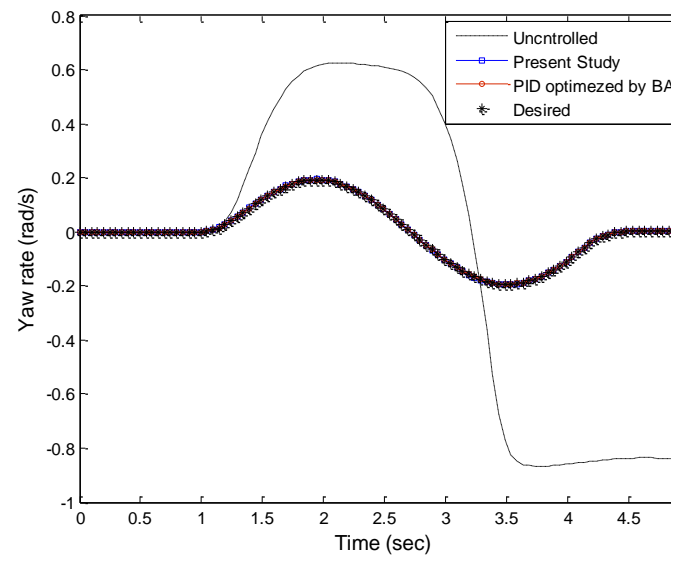

(a)

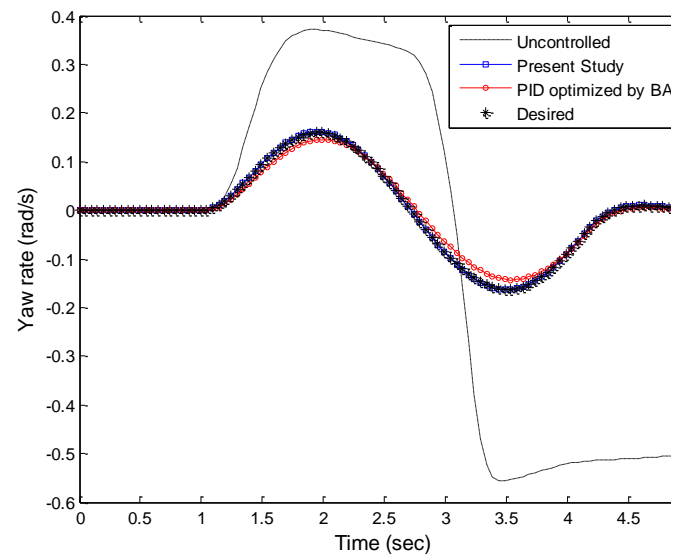

(b)

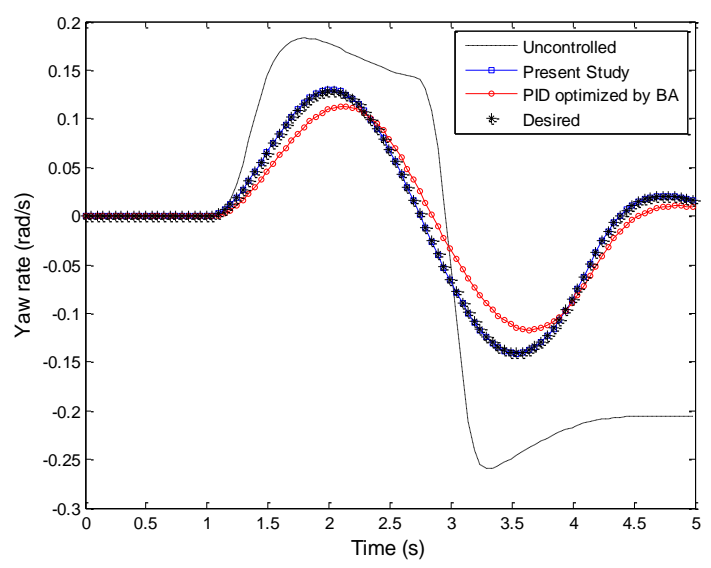

(c)

Figure 5. The desired, uncontrolled, and controlled yaw rate of the vehicle for the change lane manoeuvre when the road coefficient friction is a: $1, \mathrm{~b}: 0.6$, and c: 0.3 . 
Based on Figure 5, for the almost regular road condition i.e. Figure 5(a), both the controllers can follow the desired trajectory. It is completely expectable because the Bees Algorithm is used to find optimized controller for this road condition only. Therefore, in this condition, the coefficients of PID controller are the optimized ones, and the augmented neural network has no need to modify them. In other words, the coefficients of controller with and without applying the neural network are the same and their performances are alike. Once the road conditions change, the PID optimized only by the BA cannot completely track the desired trajectory and the use of an adaptation (such as the ANN in this work) is unavoidable. To overcome this problem, some may use a precise bank of data with large amount of calculations and simulations to interpolate the conditions and then reach the desired controller. Instead, we use the logic of trained neural networks with definitely fewer determined input/output signals to decide about the desired controller at unknown conditions [34]. Figure 5(b) and Figure 5(c) show how this proposed adaptive optimized PID controller can completely track the desired trajectories for other road conditions.

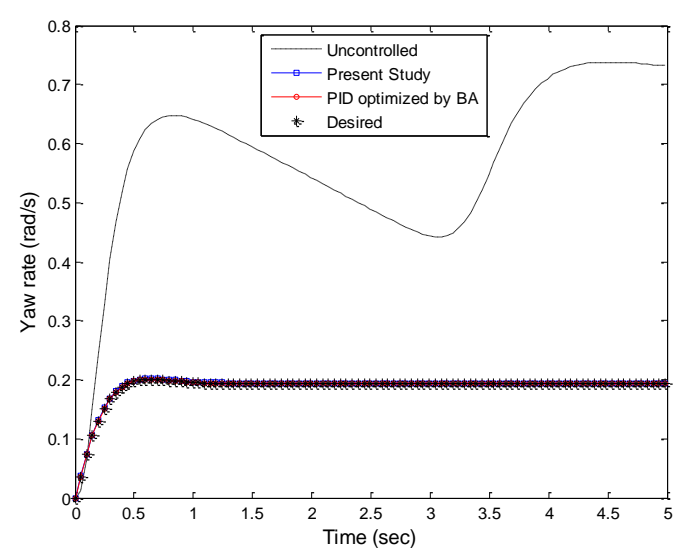

(a)

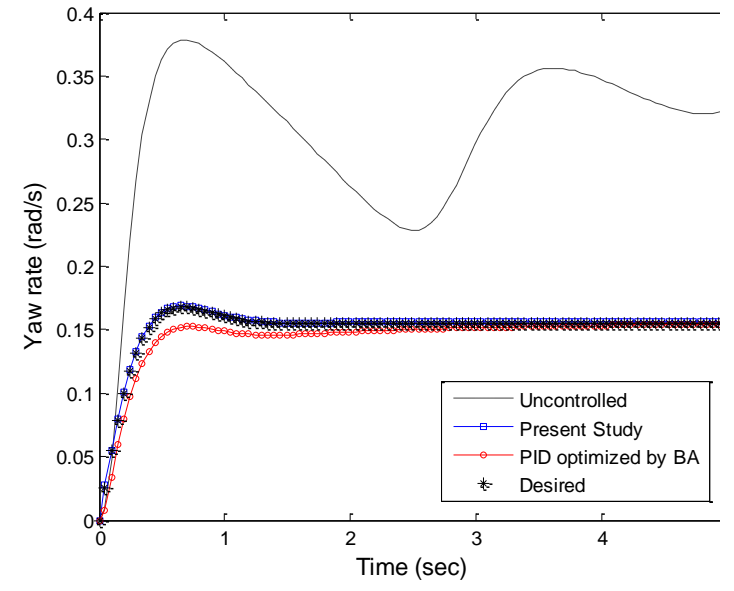

(b)

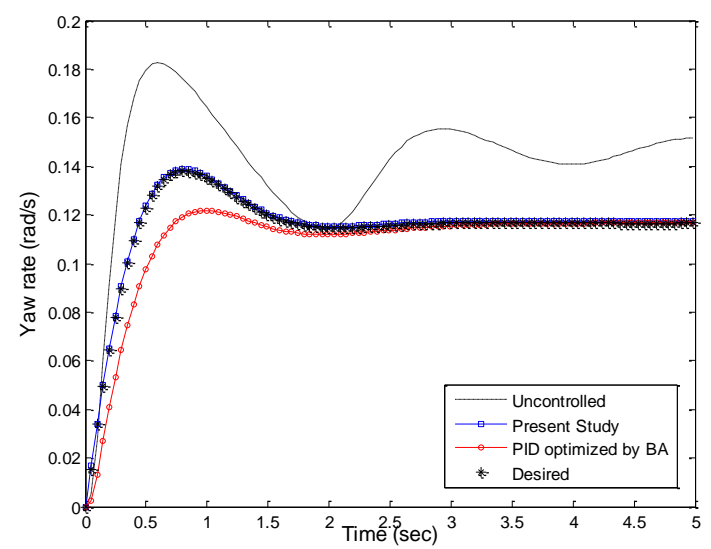

(c)

Figure 6. The desired, uncontrolled, and controlled yaw rate of the vehicle for the J-turn manoeuvre when the road coefficient friction is a: $1, b: 0.6$, and c: 0.3 .

The simulation is repeated for the other two manoeuvers, i.e. the J-turn and fishhook. Figure 6 and Figure 7 show how the optimized adapted controller that was previously designed in previous section can follow the desired yaw rate trajectory for 
different road conditions. They explain also the effectiveness of the proposed controller in this study in comparison with the one which used the BA only. To better quantify the effectiveness, the integral of squared error between the desired and actual trajectories for different manoeuvers and road conditions, are evaluated and compared to each other in Table 3. In fact, in Table 3 one can compare the performance of the controllers of cases quantitatively and entirely. With respect to this table, no differences are seen between the errors for ordinary road i.e. when the coefficient of friction equals to 1. As expressed previously, this is because the Bees Algorithm was designed and tuned for this road condition. Thus, the adjoint neural network does not have any effect on the controller. For other roads, the optimized PID controller is still good but not great. The more we move away from the reference road, the error will be larger. Thus, for more slippery roads, using the artificial neural network can help decrease the error significantly. In other words, the more slippery the road is, more adaptation needs to be applied.

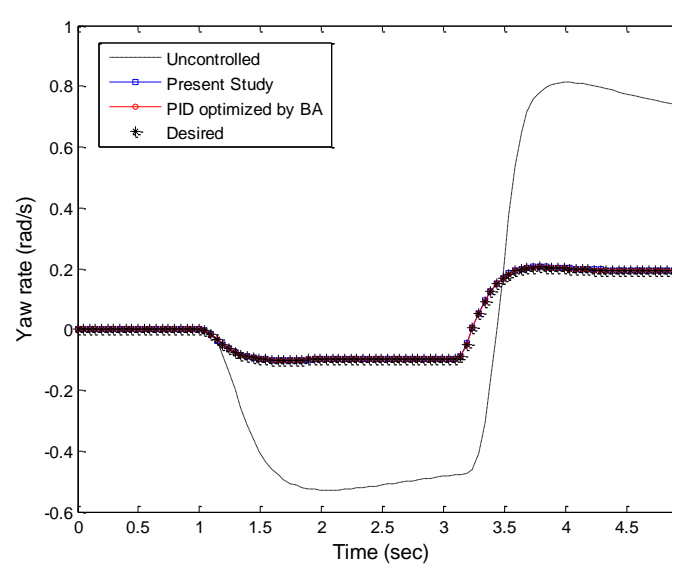

(a)

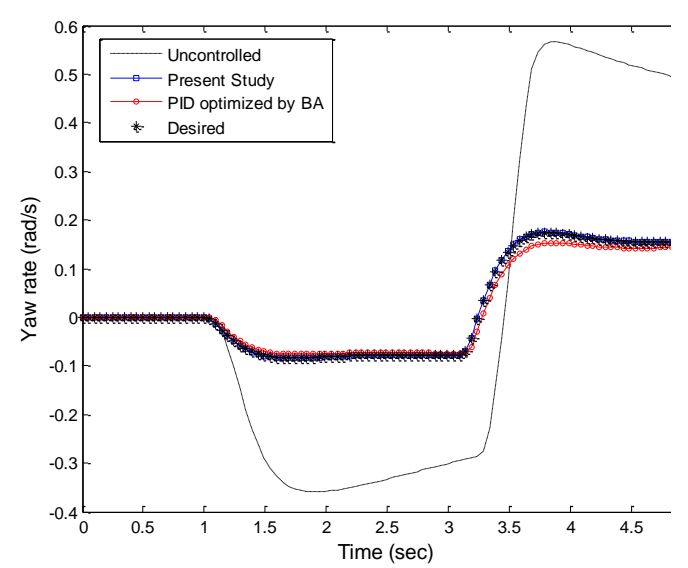

(b)

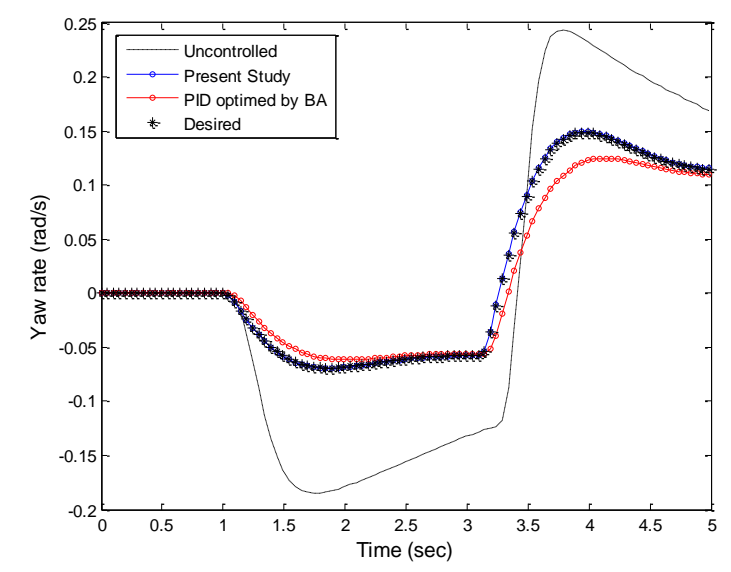

(c)

Figure 7. The desired, uncontrolled, and controlled yaw rate of the vehicle for the fishhook manoeuvre when the road coefficient friction is a: $1, \mathrm{~b}: 0.6$, and $\mathrm{c}: 0.3$. 
Table 3. The integral of squared error between the desired and actual trajectory for our case studies.

\begin{tabular}{|c|c|c|c|}
\hline $\begin{array}{l}\text { Manoeuver/ Control- } \\
\text { ler }\end{array}$ & $\begin{array}{l}\text { PID + BA + } \\
\text { ANN }\end{array}$ & $\begin{array}{l}\mathrm{PID}+ \\
\mathrm{BA}\end{array}$ & Improvement \\
\hline \multicolumn{4}{|c|}{ Coefficient of friction $=1$} \\
\hline Change lane & $1.44 \times 10^{-6}$ & $1.44 \times 10^{-6}$ & Nothing \\
\hline J-turn & $3.86 \times 10^{-6}$ & $3.86 \times 10^{-6}$ & Nothing \\
\hline Fishhook & $2.00 \times 10^{-6}$ & $2.00 \times 10^{-6}$ & Nothing \\
\hline \multicolumn{4}{|c|}{ Coefficient of friction $=0.6$} \\
\hline Change lane & $1.46 \times 10^{-6}$ & $7.6 \times 10^{-4}$ & $\begin{array}{l}\text { Reduced by } \\
524 \text { times }\end{array}$ \\
\hline J-turn & $3.95 \times 10^{-6}$ & $4.8 \times 10^{-4}$ & $\begin{array}{l}\text { Reduced by } \\
122 \text { times }\end{array}$ \\
\hline Fishhook & $2.04 \times 10^{-6}$ & $8.5 \times 10^{-4}$ & $\begin{array}{l}\text { Reduced by } \\
417 \text { times }\end{array}$ \\
\hline \multicolumn{4}{|c|}{ Coefficient of friction $=0.3$} \\
\hline Change lane & $1.08 \times 10^{-6}$ & $15.8 \times 10^{-4}$ & $\begin{array}{l}\text { Reduced by } \\
1463 \text { times }\end{array}$ \\
\hline J-turn & $2.45 \times 10^{-6}$ & $5.7 \times 10^{-4}$ & $\begin{array}{l}\text { Reduced by } \\
233 \text { times }\end{array}$ \\
\hline Fishhook & $1.35 \times 10^{-6}$ & $12.6 \times 10^{-4}$ & $\begin{array}{l}\text { Reduced by } \\
933 \text { times }\end{array}$ \\
\hline
\end{tabular}

\section{CONCLUSIONS}

In this paper, a PID controller optimized by the honey bees' algorithm, and trained by the artificial neural networks to improve the handling of vehicles in different road conditions was proposed. The presented controller can track the vehicle with acceptable performance in three famous driving manoeuvers i.e. change lane, J-turn, and fishhook in regular, slippery, and almost icy roads. The coefficient of friction is picked up in this study as an index to model the road conditions; however, the method can be applied for other unpredictable indices. To show the designed controller's effectiveness to work in different road conditions, a comparison between the behaviour of the system in the presence and absence of augmented neural network is made. No differences were seen between the squared error between the desired and actual trajectories for regular road. For slippery roads, the optimized PID controller is still good but not great. The more we move away from the regular reference road, the error will be larger. Thus, for more slippery roads, using the artificial neural network can help in decreasing the error significantly. In presence of artificial neural network, the error reduces from 122 times in J-turn manoeuvre in slippery roads to 1463 times in change-lane manoeuvre in almost icy road. The results of this work may encourage further theoretical and experimental studies in this area.

\section{ACKNOWLEDGEMENTS}

The authors would like to thanks to Hydro-Aeronautical Research Center, Shiraz University, Shiraz, 71348-13668, Iran for their institutional support. 


\section{REFERENCES}

[1] Rajamani R. Vehicle dynamics and control: Springer Science \& Business Media; 2011.

[2] Li B, Rakheja S, Feng Y. Enhancement of vehicle stability through integration of direct yaw moment and active rear steering. Proceedings of the Institution of Mechanical Engineers, Part D: Journal of Automobile Engineering. 2015:0954407015596255.

[3] Song J. Active front wheel steering model and controller for integrated dynamics control systems. International Journal of Automotive Technology. 2016;17:26572.

[4] Zulkarnain N, Zamzuri H, Mazlan S. Ride and handling analysis for an active anti-roll bar: case study on composite nonlinear control strategy. International Journal of Automotive and Mechanical Engineering. 2014;10:2122-242.

[5] Maharun M, Baharom M, Mohd M. Ride comfort simulation of a vehicle equipped with semi-active steering system. International Journal of Automotive and Mechanical Engineering. 2015;11:2495 -503.

[6] Ackermann J, Bünte T, Odenthal D. Advantages of active steering for vehicle dynamics control. 1999.

[7] Kazemi R, Bahaghighat MK, Panahi K. Yaw moment control of four wheel steering vehicle by fuzzy approach. Industrial Technology, 2008 ICIT 2008 IEEE International Conference on: IEEE; 2008. p. 1-7.

[8] Li Q, Shi G, Lin Y, Wei J. Yaw rate control of active front steering based on fuzzy-logic controller. Education Technology and Computer Science (ETCS), 2010 Second International Workshop on: IEEE; 2010. p. 125-8.

[9] Canale M, Fagiano L, Milanese M, Borodani P. Robust vehicle yaw control using an active differential and IMC techniques. Control Engineering Practice. 2007; 15:923-41.

[10] Wu S, Zhu E, Qin M, Ren H, Lei Z. Control of four-wheel-steering vehicle using ga fuzzy neural network. Intelligent Computation Technology and Automation (ICICTA), 2008 International Conference on: IEEE; 2008. p. 869-73.

[11] $\mathrm{Wu}$ J, Zhao Y, Ji X, Liu Y, Yin C. A modified structure internal model robust control method for the integration of active front steering and direct yaw moment control. Science China Technological Sciences. 2015;58:75-85.

[12] Wasim M, Kashif A, Awan AU, Khan MM, Wasif M, Ali W. H-infinity control via scenario optimization for handling and stabilizing vehicle using AFS control. Computing, Electronic and Electrical Engineering (ICE Cube), 2016 International Conference on: IEEE; 2016. p. 307-12.

[13] Wu J, Zhao Y, Ji X, Liu Y, Zhang L. Generalized internal model robust control for active front steering intervention. Chinese Journal of Mechanical Engineering. 2015;28:285-93.

[14] Jin X, Yin G, Chen J, Chen N. Robust guaranteed cost state-delayed control of yaw stability for four-wheel-independent-drive electric vehicles with active front steering system. International Journal of Vehicle Design. 2015;69:304-23. 
[15] Zhuo-Ming C, Yun-Xia W, Wei-Xin L, Zhen X, Han-Lin-Wei X. Artificial bee colony algorithm for modular neural network. International Symposium on Neural Networks: Springer; 2013. p. 350-6.

[16] RN J. Vehicle dynamics: theory and application. Springer Science \& Business Media. 2013 Nov 19.

[17] Wong JY. Theory of ground vehicles: John Wiley \& Sons; 2001.

[18] Kinjawadekar T, Dixit N, Heydinger GJ, Guenther DA, Salaani MK. Vehicle dynamics modeling and validation of the 2003 ford expedition with esc using carsim. SAE Technical Paper; 2009.

[19] Rezai M, Shirazi KH. Robust handling enhancement on a slippery road using quantitative feedback theory. Proceedings of the Institution of Mechanical Engineers, Part D: Journal of Automobile Engineering. 2014:0954407013518037.

[20] Seeley TD. The wisdom of the hive: the social physiology of honey bee colonies: Harvard University Press; 2009.

[21] Camazine S. Self-organization in biological systems: Princeton University Press; 2003.

[22] Pham D, Ghanbarzadeh A, Koc E, Otri S, Rahim S, Zaidi M. The Bees Algorithm. Tech. Note. Manufacturing Eng Centre, Cardiff University, UK. 2005.

[23] Pham DT, Soroka AJ, Koc E, Ghanbarzadeh A, Otri S. Some applications of the bees algorithm in engineering design and manufacture. ICMA; 2007.

[24] Pham D, Ghanbarzadeh A. Multi-objective optimisation using the bees algorithm. 3rd International Virtual Conference on Intelligent Production Machines and Systems (IPROMS 2007): Whittles, Dunbeath, Scotland2007. p. 111-6.

[25] Pham D, Soroka AJ, Ghanbarzadeh A, Koc E, Otri S, Packianather M. Optimising neural networks for identification of wood defects using the bees algorithm. 2006 4th IEEE International Conference on Industrial Informatics: IEEE; 2006. p. 1346-51.

[26] Yuce B. Novel computational technique for determining depth using the Bees Algorithm and blind image deconvolution: Cardiff University; 2012.

[27] Pham D, Ghanbarzadeh A, Koc E, Otri S, Rahim S, Zaidi M. The bees algorithma novel tool for complex optimisation. Intelligent Production Machines and Systems-2nd I PROMS Virtual International Conference (3-14 July 2006)2011.

[28] Luo J, Wang Q, Fu L. Application of modified artificial bee colony algorithm to flatness error evaluation. Guangxue Jingmi Gongcheng(Optics and Precision Engineering). 2012;20:422-30.

[29] Diwold K, Beekman M, Middendorf M. Honeybee optimisation-an overview and a new bee inspired optimisation scheme. Handbook of swarm intelligence: Springer; 2011. p. 295-327.

[30] Karaboga D. An idea based on honey bee swarm for numerical optimization. Technical report-tr06, Erciyes university, engineering faculty, computer engineering department; 2005.

[31] Fullér R. Neural fuzzy systems. 2013.

[32] McCulloch WS, Pitts W. A logical calculus of the ideas immanent in nervous activity. The bulletin of mathematical biophysics. 1943;5:115-33.

[33] Hebb DO. The organization of behavior: A neuropsychological theory: Psychology Press; 2005.

[34] Psaltis D, Sideris A, Yamamura A. A multilayered neural network controller. IEEE control systems magazine. 1988;8:17-21. 
[35] Lin Y, Tang P, Zhang W, Yu Q. Artificial neural network modelling of driver handling behaviour in a driver-vehicle-environment system. International Journal of Vehicle Design. 2005;37:24-45.

[36] Zadeh AG, Fahim A, El-Gindy M. Neural network and fuzzy logic applications to vehicle systems: literature survey. International Journal of Vehicle Design. 1997;18:132-93.

[37] Rojas R. The backpropagation algorithm. Neural networks: Springer; 1996. p. 149-82.

[38] Werbos P. Beyond regression: New tools for prediction and analysis in the behavioral sciences. 1974.

[39] Rumelhart DE, Hinton GE, Williams RJ. Learning representations by backpropagating errors. Cognitive modeling. 1988;5:1.

[40] Kriesel D. A brief Introduction on Neural Networks. 2007.

\section{NOMENCLATURE}

$\begin{array}{cl}C_{f}, & \text { Cornering stiffness of the front wheels } \\ C_{r} & \text { Cornering stiffness of the rear wheels } \\ F_{c r} & \text { Lateral forces of rear wheels } \\ F_{c f} & \text { Lateral forces front wheels } \\ l & \text { The distance between the rear and front wheels } \\ \mathrm{m} & \text { The total mass of car, } \\ m_{f} & \text { The equivalent front mass } \\ m_{r} & \text { The equivalent rear mass } \\ I_{z} & \text { The mass moment of inertia } \\ v_{y} & \text { Velocity along } y \text { direction } \\ v_{x} & \text { Velocity along } x \text { direction } \\ \delta_{f} & \text { The steering angle of front wheel } \\ \psi & \text { The car yaw angle. } \\ \alpha_{f} & \text { The lateral slip angle of front wheel } \\ \alpha_{r} & \text { The lateral slip angle of rear wheel } \\ \beta & \text { The sideslip angle of car } \\ 4 W D & \text { 4-wheel-differential } \\ \text { AFS } & \text { Active front steering } \\ \text { ANN } & \text { Artificial Neural Network } \\ \text { BA } & \text { Bees Algorithm } \\ \text { NPQL } & \text { Nonlinear programming quadratic line search } \\ \text { PID } & \text { Proportional-integral-derivative } \\ \text { RSM } & \text { Response surface model }\end{array}$

